\title{
A Reflection on the Use of Enoxaparin Based on Three Case Reports
}

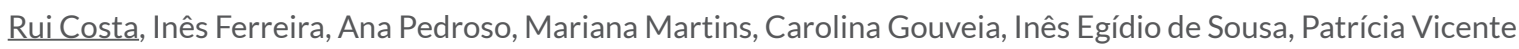 \\ Internal Medicine Department, Hospital São Francisco Xavier, Lisbon, Portugal
}

Doi: 10.12890/2018_001000 - European Journal of Case Reports in Internal Medicine - ๑ EFIM 2018

Received: $21 / 11 / 2018$

Accepted: $23 / 11 / 2018$

Published: $10 / 12 / 2018$

How to cite this article: Costa R, Ferreira I, Pedroso A, Martins M, Gouveia C, Egidio de Sousa I, Vincente P. A reflection of the use of enoxaparin based on three case reports. EJCRIM 2018;5: doi:10.12890/2018_001000.

Conflicts of Interests: The Authors declare that there are no competing interests.

This article is licensed under a Commons Attribution Non-Commercial 4.0 License

\section{ABSTRACT}

Enoxaparin is indicated for the treatment or prevention of many clinical disorders including deep vein thromboembolism, atrial fibrillation and mechanical valve thrombosis. It is one of the most commonly prescribed drugs in hospitals. However, haemorrhagic complications can occur, particularly in the elderly, patients with renal function impairment and patients with a very high or very low body weight. The authors describe the cases of three patients who had one or more risk factors for haemorrhagic complications, such as abdominal haematomas. The clinical presentation was similar in all three cases, with sudden-onset abdominal pain, an altered state of consciousness and hypotension. In all cases, investigation showed acute anaemia and large abdominal haematomas on imaging studies. A conservative approach was taken in the three patients, with suspension and reversal of anticoagulation, fluid resuscitation and red blood cell transfusion. Haemodynamic stability was achieved in two of the patients, but the third patient died.

The authors consider it is important to present these case reports because of the widespread use of enoxaparin, and the need for rigorous dose adjustment for renal function variations and body weight. We hope this article raises awareness of haemorrhagic complications in high-risk groups and propose protocols are introduced for dose adjustment and monitoring the efficacy of enoxaparin.

\section{LEARNING POINTS}

- Clinicians should consider the possibility of enoxaparin-associated haemorrhagic complications in high-risk groups.

- Prompt identification and treatment of the haemorrhagic disorder can improve outcome.

- Correct dosing with anti-Xa in high-risk populations, such as the elderly, those with abnormal renal function and those with extremely high or low body weight, may be helpful in patients anticoagulated with enoxaparin.

\section{KEYWORDS}

Enoxaparin, spontaneous hematomas, anticoagulation side effects

\section{CASE REPORT}

\section{Case 1}

An 81-year-old woman, with a medical history of obesity and atrial fibrillation anticoagulated with warfarin, was admitted to a stroke unit due to a right cerebellar infarction of cardioembolic aetiology resulting from an INR below the therapeutic range. Due to the size and location of the infarction, anticoagulation was re-initiated on the 5 th day with enoxaparin $80 \mathrm{mg}$ twice daily. On the 8th day of hospitalization, the patient had a tonic-clonic seizure because of a temporary 16 -second sinoatrial pause, after which she developed right quadrant abdominal pain associated with hypotension. An abdominal CT scan showed a right rectus abdominis muscle haematoma measuring $12 \times 7 \times 7 \mathrm{~cm}$. Haemoglobin $(\mathrm{Hb})$ dropped from $12.4 \mathrm{~g} / \mathrm{dl}$ to $7.4 \mathrm{dl}$, while the coagulation profile (activated partial prothrombin time (aPTT), thrombin time 
(TP) and INR), platelets and renal function were normal. Anticoagulation was suspended, and the patient was stabilized with crystalloid solutions, 5 units of red blood cells (RBC) and four units of prothrombin complex concentrates (PCC), with recovery of haemodynamic stability and $\mathrm{Hb}$ values. Surgical intervention was not performed due to clinical improvement following conservative measures. CT scan re-evaluation on the following days revealed the size of the haematoma was increasing $(24.5 \times 13.7 \times 6.5 \mathrm{~cm})$ and the appearance of a pelvic haematoma measuring $14.6 \times 7.4 \mathrm{~cm}$ (Figs. 1 and 2). The patient was discharged after a 52-day hospital stay, with anticoagulation suspended.

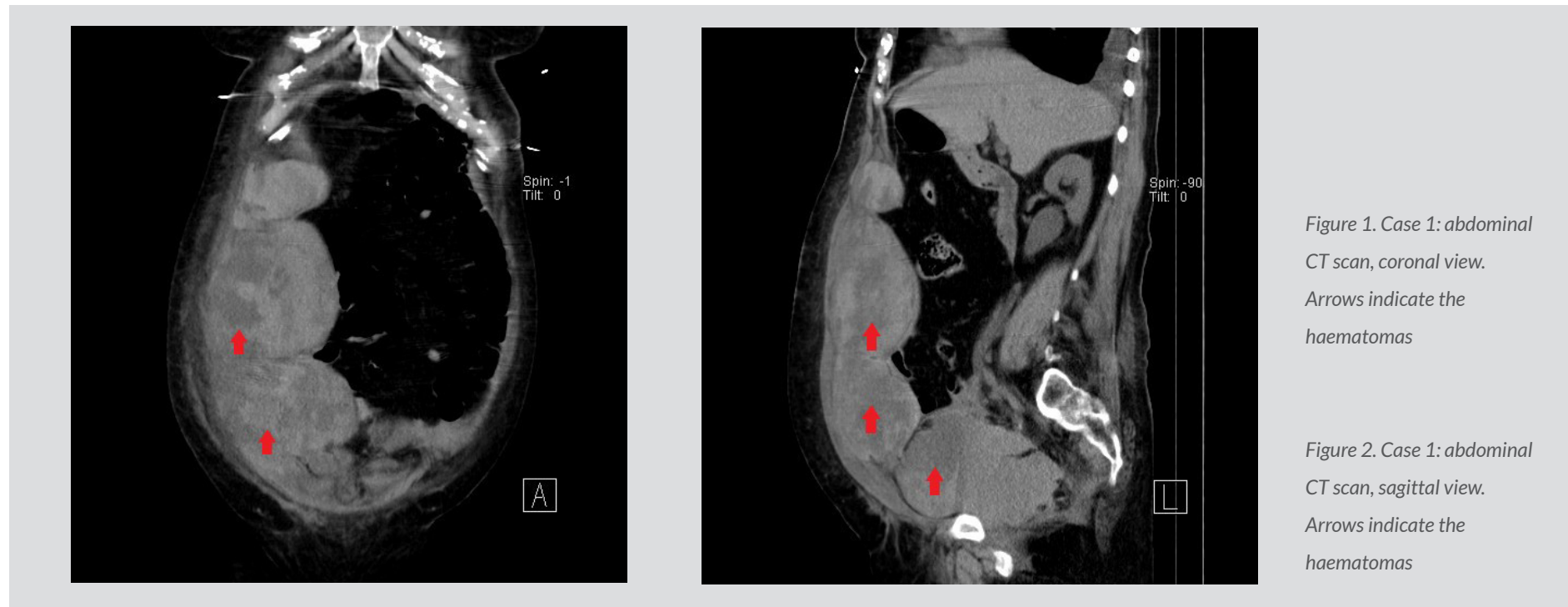

Case 2

A 66-year-old man, with a diagnosis of atrial fibrillation anticoagulated with warfarin, and symptomatic aortic stenosis (NYHA functional class III), was admitted to a stroke unit following a thalamic haemorrhagic stroke. His INR was in the therapeutic range (2.4) and anticoagulation was suspended. Despite slow neurological improvement, the patient's hospital stay was complicated by cardiorenal syndrome type 1 and nosocomial respiratory infection which delayed discharge. After stabilization, a re-evaluation CT scan showed total reabsorption of the haematoma, and after discussion with the neurosurgery department, anticoagulation was resumed with enoxaparin 60 mg twice daily adjusted for a glomerular filtration rate (GFR) of $42 \mathrm{ml} / \mathrm{min}$. Five days later, the patient developed symptomatic hypotension after sitting on a chair, with mucocutaneous pallor but no other relevant signs on examination. Laboratory results revealed an acute drop in $\mathrm{Hb}$ from 8.9 $\mathrm{g} / \mathrm{dl}$ to $5.8 \mathrm{~g} / \mathrm{dl}$, a GFR of $42 \mathrm{ml} / \mathrm{min}$ and normal platelet numbers and coagulation profile (INR, aPTT, PT). Anticoagulation was suspended, and fluid resuscitation with crystalloids, 4 units of RBC and three units of PCC was administered. An abdominal and pelvic CT scan was performed and showed large retroperitoneal haematomas, the largest measuring $40 \times 9 \mathrm{~cm}$ (Figs. 3 and 4). The case was discussed with the vascular surgery department and surgical intervention was rejected. The patient was later transferred to an intensive care unit where he died soon afterwards.
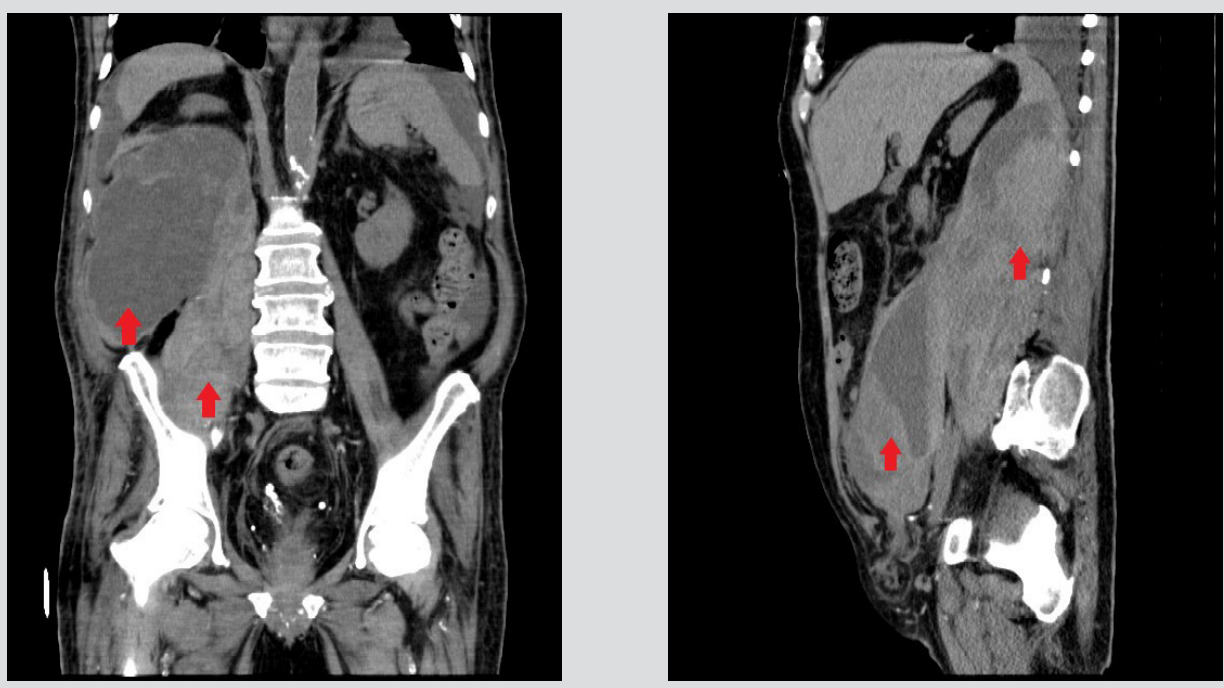

Figure 3. Case 2: abdominal CT scan, coronal view. Arrows indicate the haematomas

Figure 4. Case 2: abdominal CT scan, sagittal view. Arrows indicate the haematomas 
Case 3

An 84-year-old woman was admitted to the orthogeriatric unit for surgical intervention for a traumatic malleolar fracture without complications. Atrial fibrillation was diagnosed during the first few days of her stay and enoxaparin was started at a dose of $60 \mathrm{mg}$ twice daily for a body weight of $65 \mathrm{~kg}$ and GFR $37 \mathrm{ml} / \mathrm{min}$. Over the next 4 days, the patient complained of diffuse abdominal discomfort, with a tendency to hypotension and tachycardia. On examination she had mucous pallor, and slight discomfort on hypogastric palpation. Hb values decreased gradually from $10.5 \mathrm{~g} / \mathrm{dl}$ to $5 \mathrm{~g} / \mathrm{dl}$. A nasogastric tube was placed without signs of upper gastrointestinal bleeding. Rectal examination was performed without findings suggestive of lower GI bleeding. An abdominopelvic CT scan showed a retroperitoneal haematoma measuring $18 \times 10 \times 6 \mathrm{~cm}$ with signs of recent haemorrhage (Figs. 5 and 6). Enoxaparin was suspended, and 4 units of RBC and four-factor PCC were administered. On the following days, due to the increasing size of the haematoma, the case was discussed with the surgical department but intervention was rejected.

The rest of the patient's hospital stay was made more difficult by metabolic and infectious complications, and prolonged in particular because of social issues. The patient was discharged 107 days after admission, with anticoagulation suspended.
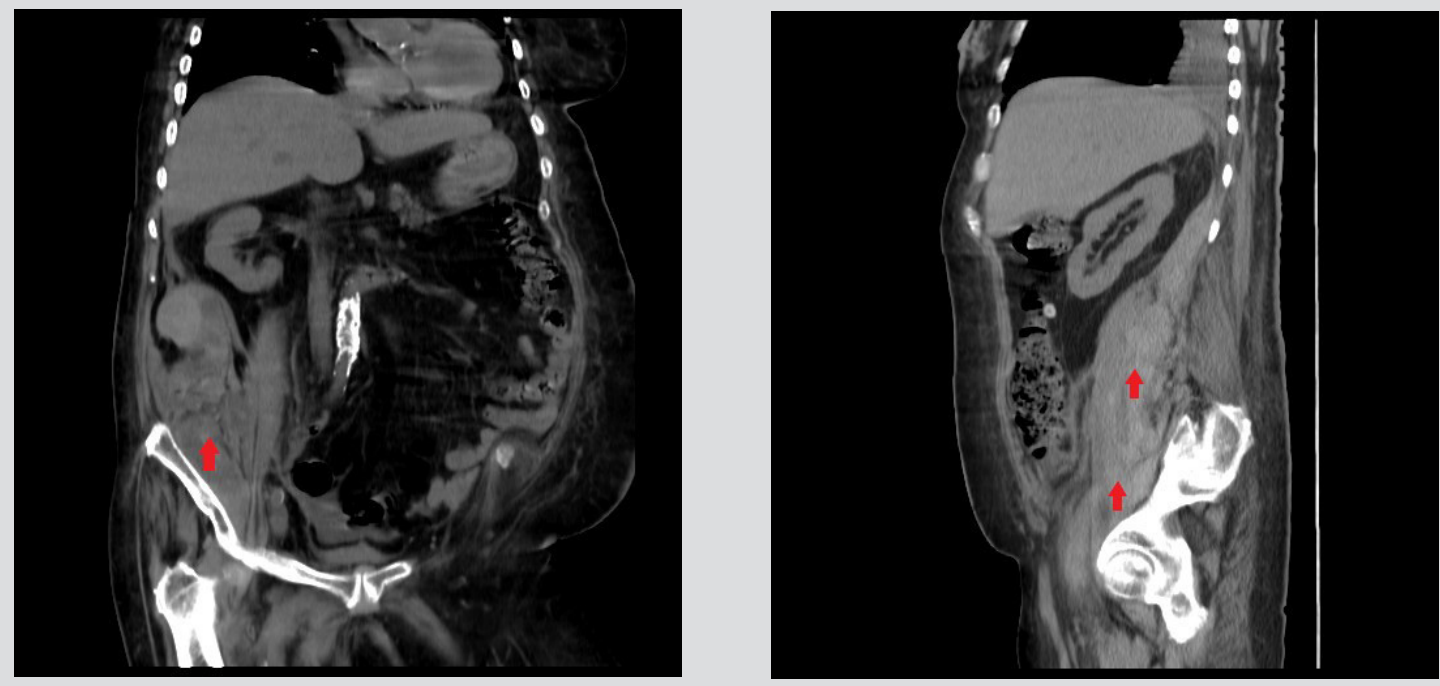

Figure 5. Case 3: abdominal CT scan, coronal view. The arrow indicates the haematoma

Figure 6. Case 3: abdominal CT scan, coronal view. Arrows indicate the haematoma

\section{DISCUSSION}

Enoxaparin is a low-molecular-weight heparin indicated for the treatment or prevention of multiple clinical disorders, including deep vein thrombosis, pulmonary embolism, ST elevation myocardial infarction, stroke prevention in atrial fibrillation, and mechanical valve thrombosis. It is one of the most commonly prescribed drugs in hospitals, having widely replaced unfractionated heparins due to its more predictable pharmacokinetic profile and longer plasma half-life. It acts by binding to antithrombin III, forming a complex that inactivates factor Xa.

Haemorrhagic complications are known but not often reported. It is believed that the underlying mechanism is heparin-induced immune microangiopathy, and that complications are more common in particular groups such as the elderly, patients with abnormal renal function and those with very high or very low bodyweight. Even with early detection and prompt treatment, enoxaparin-associated haemorrhage mortality can range from $4 \%$ to $20 \%$ in reported series ${ }^{[1]}$.

In the clinical cases described here, the patients had one or more factors associated with an increased risk of haemorrhagic complications ${ }^{[2]}$. The clinical presentation was fairly common in all cases, with sudden onset of abdominal pain, altered state of consciousness and associated hypotension. All patients had acute drops of 3.1-5 g/dl in Hb levels.

A conservative approach to treatment was taken in all cases, with suspension and reversal of anticoagulation, which resulted in haemodynamic stability in case 1. In case 2, and for future approaches, referral to a centre with experience in angiography and arterial embolization could have been an option, as it can be beneficial in cases refractory to conservative treatment ${ }^{[3]}$. However, due to haemodynamic instability and rapid clinical deterioration, this referral was not an option at the time. In case 3, the insidious clinical presentation over 3-4 days made it more difficult to diagnose the acute haemorrhage. Retroperitoneal localization likely provides fewer findings on clinical examination, demonstrating the importance of actively considering this possibility in anticoagulated patients with acute or subacute anaemia. 


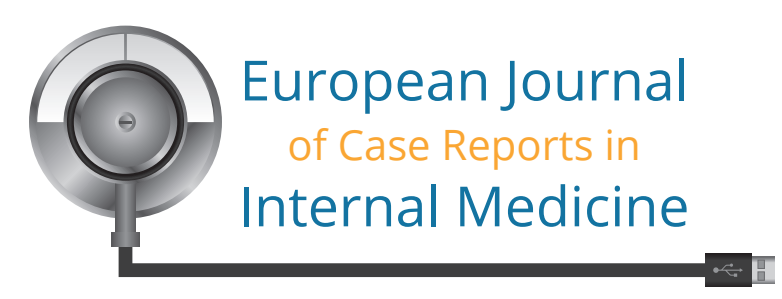

The authors consider it is important to share these clinical cases because of the widespread use of enoxaparin, sometimes without the advantages and disadvantages being properly considered. Renal function dose adjustments should be revised continuously during hospital stays due to the variability of the GFR, and body weight must always be taken into account for dosing determination.

We want to raise awareness of these unfamiliar haemorrhagic complications in high-risk groups and propose the use of dose adjustments and protocols for monitoring the efficacy of anticoagulation with anti-factor Xa. However, large, prospective clinical trials are needed to study these proposed measures in greater detail. We would also like to highlight the importance of early identification and multidisciplinary decision-making with surgical and interventional radiology teams, in order to improve outcome in these patients.

\section{REFERENCES}

1. Carkman S, Ozben V, Zengin K, Somuncu E, Karatas A. Spontaneous rectus sheath hematomas: an analysis of 15 cases. Ulus Travma Acil Cerrahi Derg 2010;16:532-536.

2. Cestac P, Bagheri H, Lapeyre-Mestre M, Sié P, Fouladi A, Maupas E, Léger P, Fontan B, Massip P, Montastruc JL. Utilisation and safety of low molecular weight heparins: prospective observational study in medical inpatients. Drug Saf 2003;26:197-207.

3. Rimola J, Perendreu J, Falcó J, Fortuño JR, Massuet A, Branera J. Percutaneous arterial embolization in the management of rectus sheath hematoma. AJR Am J Roentgenol 2007;188:W497-W502. 